\title{
CONE BEAM COMPUTED TOMOGRAPHY REVEALS ALTERED TRABECULAR BONE STRUCTURE IN ACROMEGALY
}

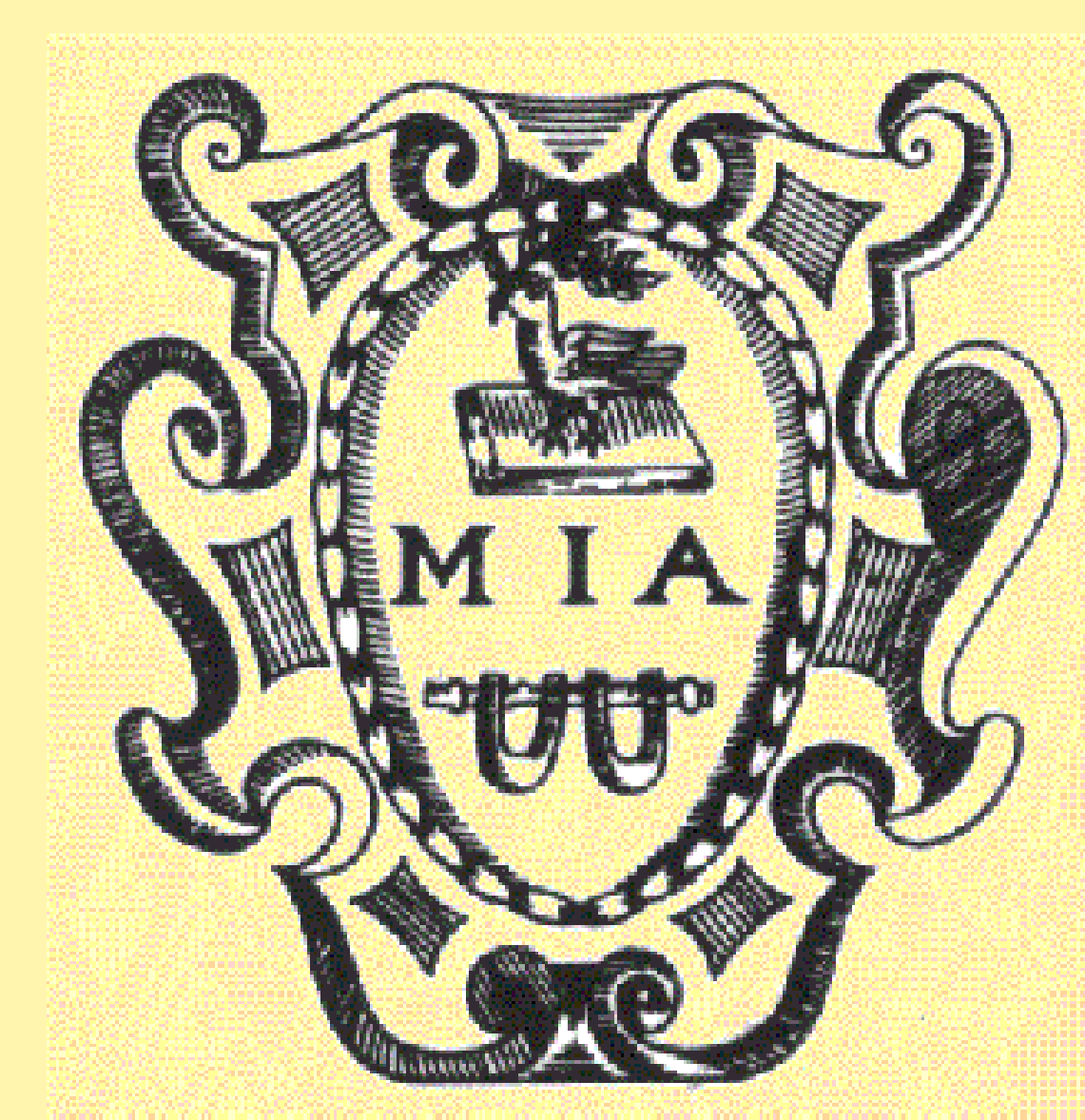

\author{
A. Giustina ${ }^{1}$, G. Mazziotti ${ }^{1}$, M. Maddalo², F. Maffezzoni ${ }^{1}$, S. Frara ${ }^{1}$, I. Zorza², P. Soldini² ${ }^{2}$ F. Doglietto ${ }^{3}$, R. Maroldi². \\ ${ }^{1}$ Endocrinology, ${ }^{2}$ Radiology and ${ }^{3}$ Neurosurgery, University of Brescia, Italy.
}

\section{Background}

Over the last years, there has been evidence that acromegaly may cause skeletal fragility with high risk of vertebral fractures. The diagnostic approach to this complication is still a matter of uncertainty, since DXA measurement of bone mineral density (BMD) does not provide reliable information on fracture risk in acromegaly.

The cone beam computed tomography (CBCT) technique offers three-dimensional images for the radiographed skeletal area with the advantage to use non-dedicated equipments as compared to the peripheral Quantitative Computed Tomography.

\section{Aim}

To investigate whether a non-dedicated CBCT system may provide information on the skeletal abnormalities caused by acromegaly.

\section{Patients and Controls}

\section{Inclusion Criteria}

1. Diagnosis of acromegaly (only for patients);

2. Age older than 18 years;

3. Informed consent.

\section{Exclusion Criteria}

1. Neoplastic diseases in progression;

2. Treatment with anti-osteoporotic drugs except for calcium and vitamin D;

3. Treatment with drugs causing osteoporosis except for those used to replace hypopituitarism;

4. Prolonged immobilization;

5. Trauma;

6. Surgical intervention on the spine;

7. Pregnancy.

Table 1: Demographical and clinical features of patients with acromegaly (pts.) and control subjects enrolled in the study. ${ }^{*}, p<0.05$ vs acromegaly pts.

\begin{tabular}{|lcc|}
\hline & Acromegaly pts & Controls \\
\hline Cases & 22 & 16 \\
\hline Sex (F/M) & $12 / 10$ & $6 / 10$ \\
\hline Age (yrs) & $62(25-72)$ & $59(25-68)$ \\
\hline BMI (Kg/m2) & $28(20-36)$ & $27(22-32)$ \\
\hline Serum IGF-I (ng/mI) & $187(115-1387)$ & - \\
\hline Active acromegaly (cases) & $11(50.0 \%)$ & - \\
\hline Duration of active acromegaly (months) & $24(6-112)$ & - \\
\hline Neurosurgery (cases) & $16(72.8 \%)$ & - \\
\hline Somatostatin analogs therapy & $19(86.4 \%)$ & - \\
\hline Pegvisomant therapy & $1(4.5 \%)$ & - \\
\hline Radiotherapy & 0 & $5(22.7 \%)^{*}$ \\
\hline Untreated hypogonadism & & 0 \\
Post-menopausal females & $11(50.0 \%)$ & 0 \\
\hline $\begin{array}{l}\text { Pre-menopausal females } \\
\text { Males }\end{array}$ & $1(4.5 \%)$ & $0^{*}$ \\
\hline Treated hypoadrenalism & 0 & $0^{*}$ \\
\hline Treated hypothyroidism & $1(4.5 \%)$ & - \\
\hline F, fel & $9(40.9 \%)$ & \\
\hline
\end{tabular}

F, females: M, males; yrs, years; BMI, body mass index

\section{Protocol}

Geometric trabecular parameters and cortical thickness were measured at the distal radius using a high resolution CBCT system (Newtom 5G; QR, Verona, Italy) with an isotropic voxel size of 75 microns. For each patient a volume of 120 slices was obtained, corresponding approximately to a section of $9 \mathrm{~mm}$ in the axial plane. The first CBCT slice was obtained $9.5 \mathrm{~mm}$ proximal to the endplate of the radius.

After measurement of the cortical thickness, an irregular volume of interest (VOI) containing only trabecular bone was defined through segmentation and exclusion in every slice of the cortical cross-sectional area. A fixed threshold value equal for every patient was applied in order to separate trabeculae from bone marrow in the VOI. Bone volume fraction (BV/TV), mean trabecular thickness (Th.mean) and mean trabecular separation (Sp.mean) were measured by means of BoneJ plug-in for ImageJ software version 1.47v (Rasband, W.S., ImageJ, U.S. National Institutes of Health, Bethesda, MD, USA).

All patients with acromegaly were evaluated for BMD at lumbar spine, total hip, femoral neck and distal radius using DXA machine (Lunar Prodigy 8743 , GE Medical Systems, Madison WY, USA).

\section{Results}

Patients with acromegaly showed lower trabecular bone volume fraction (BV/TV) (Fig. 1a) and mean trabecular thickness (Fig. 1b) and greater mean trabecular separation (Sp.mean) (Fig. 1c), without significant difference in cortical thickness (Fig. 1d) as compared to the control subjects.

In acromegaly patients, the duration of active disease significantly correlated with BV/TV ( $r:-0.69 ; p=0.001)$, Th.mean $(r:-0.46 ; p=0.05)$ and $S p$.mean ( $r$ : $0.47 ; p=0.04)$. In acromegaly patients, the BMD at distal radius significantly correlated with cortical thickness $(r=0.56 ; p=0.007)$, whereas the $B M D$ at either skeletal site was not significantly correlated with the trabecular parameters.
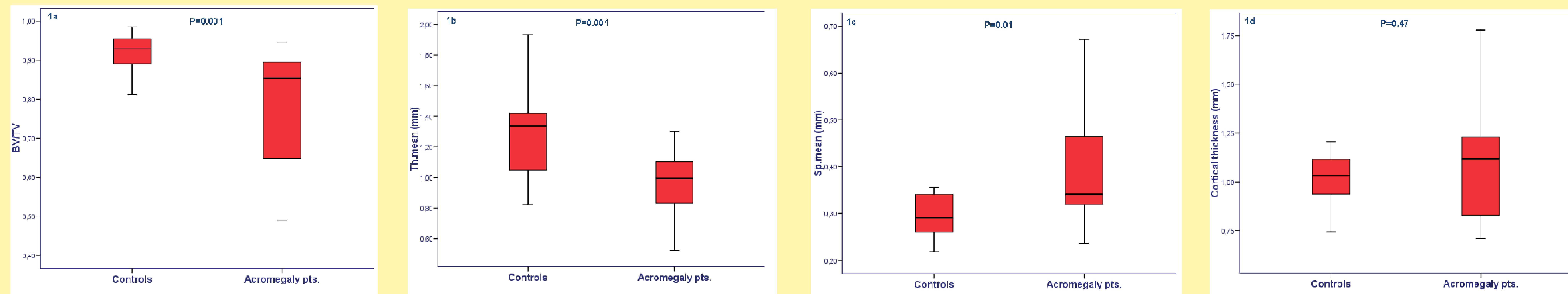

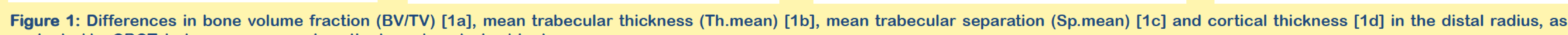
evaluated by CBCT, between acromegaly patients and control subjects.

\section{Conclusions}

In acromegaly patients, the distal radius CBCT reveals significant alterations of physiological trabecular bone structure which may be responsible for their high risk of fragility fractures even in the presence of normal BMD. 